\title{
Simulação de cenários para avaliação bioeconômica de sistemas a pasto de recria- engorda de bovinos de corte no Sul do Brasil
}

Scenario simulation for bioeconomic evaluation in stocker-finish in beef cattle grazing systems in South Brazil

\author{
Simulación de escenarios para la evaluación bioeconómica de sistemas a pasto de recria y engorde \\ de bovinos de corte em el Sur de Brasil
}

Recebido: 14/03/2021 | Revisado: 21/03/2021 | Aceito: 13/09/2021 | Publicado: 14/09/2021

Leonardo Rocha da Silva
ORCID: https://orcid.org/0000-0003-1169-993X
Universidade Federal de Pelotas, Brasil
E-mail: sispec@ @granadoegordo.com.br
Vinícius do Nascimento Lampert
ORCID: https://orcid.org/0000-0002-2907-2871
E-mail: vinicius.lampert@embrapa.br
Joal Brazzale Leal
Empresa Brasileira de Pesquisa Agropecuária, Pecuála Sul, Brasil
ORCID: https://orcid.org/0000-0002-9396-3687
Empresa Brasileira de Pesquisa Agropecuária, Pecuária Sul, Brasil
E-mail: joal.leal @embrapa.br
Alvim Faria
ORCID: https://orcid.org/0000-0002-0661-9408
Centro Universitário da Região da Campanha, Brasil
E-mail: alvimfaria@ hotmail.com
Ricardo Zambarda Vaz
ORCID: https://orcid.org/0000-0003-4505-1277
Universidade Federal de Santa Maria, Brasil
E-mail: rzvaz@ terra.com.br

\begin{abstract}
Resumo
O objetivo desse estudo foi avaliar 27 cenários simulados com dados reais e auxílio de planilhas integradas do Microsoft Excel®, para sistemas a pasto de recria-engorda de bovinos. Os cenários formaram-se a partir de três níveis de intensificação (módulos de manejo): baixo (campo nativo - $\mathrm{CN}$ ), intermediário $(\mathrm{CN}+$ pastagem de inverno - PI) e alto $(\mathrm{CN}+\mathrm{PI}+$ pastagem de verão - PV); três categorias animais: bezerro, novilho e vaca; e três arranjos comerciais conforme preços de compra/venda: alto/baixo (desfavorável), médio/médio (médio) e baixo/alto (favorável). Com preços desfavoráveis apenas o bezerro respondeu positivamente à intensificação do módulo de manejo, enquanto novilho e vaca perderam margem do módulo intermediário para o alto. A vaca apresentou margens negativas em todos os módulos de manejo. O novilho apresentou a melhor margem/área nos módulos baixo e intermediário, e o bezerro em módulo alto. Em conjuntura de preços médios, apesar de resultados agora positivos, a vaca mantém decréscimo de margem no módulo alto. O novilho apresenta ganhos crescentes à intensificação, demonstrando melhor desempenho que o bezerro em todos os módulos de manejo. Em preços favoráveis, todas as categorias apresentam aumento de margem/área à intensificação. Em módulo intermediário, a vaca apresentou a melhor margem/área entre as categorias, ao passo que em baixo e alto o novilho respondeu com a melhor margem/área. Ao avaliar a margem/cabeça/ano, em qualquer conjuntura comercial e categoria animal, o módulo alto perde competitividade em relação ao módulo intermediário. Entretanto, quando avaliada a margem/área, apenas em 3 dos 27 cenários ocorreu decréscimo da margem.
\end{abstract}

Palavras-chave: Categoria animal; Rentabilidade; Tomada de decisão.

\begin{abstract}
The aim of this study was to evaluated twenty-seven scenarios simulated in stocker-finishing beef cattle grazing systems, performed with real data and the aid of integrated Microsoft Excel ${ }^{\circledR}$ spreadsheets. The scenarios, consisted of three intensification levels (management modules): low (native pastures - NP), intermediate (NP + winter pasture $\mathrm{WP}$ ) and high (NP + WP + summer pasture - SP); three animal categories: calf, steer and cow; and three commercial arrangements, differing in the purchase and sale prices of animals: high/low (unfavorable), medium/medium (medium) and low/high (favorable). With unfavorable prices only the calf responded positively to the increasing level of system intensification, while steer and cow lost margin when the system was intensified with SP compared to the
\end{abstract}


intermediate level. Between categories, the cow has negative margins at all intensification levels, the steer has the best margin at low and intermediate levels, and the calf at high level. In the context of average prices, the cow continues to decrease its margin due to intensification with SP. However, it obtains positive results in all management modules. In this case, the steer shows increasing gains with system intensification, demonstrating better performance than the calf in all management modules. In favorable price situations, all categories show increasing gains per area as the system intensifies. At this juncture, at an intermediate intensification level, the cow has the best performance per area between categories, while at low and high levels the steer responds with the best margin. When assessing the margin/head/year, in any commercial and animal category, intensification with SP loses competitiveness in relation to the intermediate intensification management module. However, when the margin/area was evaluated, only 3 of 27 scenarios decreased the margin.

Keywords: Animal category; Decision-making; Profitability.

\section{Resumen}

Fueron simulados 27 escenarios, con datos reales y utilizando las planillas integradas del Microsoft Excel®, para sistemas a pasto de recría y engorde de bovinos. Los escenarios se conformaron a partir de tres niveles de intensificación (módulos de manejo): bajo (pastizales naturales $-\mathrm{PN}$ ), medio ( $\mathrm{PN}+$ pastizales invernales $-\mathrm{PI}$ ) y alto $(\mathrm{PN}+\mathrm{PI}+$ pastizales de verano - PV); tres categorías de animales: ternero, novillo y vaca; y tres posibles escenarios comerciales conforme a los precios de compra/venta: alto/bajo (desfavorable), medio/medio (medio) y bajo/alto (favorable). Con precios desfavorable solamente el ternero respondió positivamente a la intensificación del módulo de manejo, mientras que el novillo y la vaca perdieron margen del medio para el alto. En la coyuntura de precios medios, que, con los resultados ahora positivos, la vaca mantiene la disminución del margen en el módulo alto. En este caso, el novillo presenta ganancias crecientes conforme a la intensificación, demostrando mejor desempeño que el ternero en todos los módulos de manejo. En precios favorables, todas las categorías presentaron aumento de margen/área a la intensificación. En esta coyuntura, en el módulo medio, la vaca presentó el mejor margen/área de las categorías, mientras que, en el bajo y el alto, el novillo respondió con el mejor margen/área. Al evaluar el margen/cabeza/año, en todas las coyunturas comerciales y categorías de animales, el módulo alto pierde competitividad en relación al módulo medio. Entre tanto, cuando evaluado el margen/área, solamente en 3 de los 27 escenarios ocurrió disminución del margen.

Palabras clave: Categoría animal; Rentabilidad; Toma de decisiones.

\section{Introdução}

Os sistemas de produção de bovinos de corte têm sido economicamente pressionados e constantemente desafiados a evoluir (Freitas et al., 2017). A atual, crescente e necessária demanda por eficiência no uso da terra, estimulada pela maior competição entre as atividades agropecuárias, torna imperativa a profissionalização da pecuária de corte. Diversas pesquisas já comprovaram a aplicabilidade de inúmeras alternativas tecnológicas, sejam elas em insumos ou processos, para aumentar a produtividade dos sistemas pecuários. O conhecimento disponível atualmente é passível de realizar transformações produtivas significativas na pecuária de corte brasileira. Entretanto, a maior parte desses estudos não aborda a economicidade das alternativas produtivas nos sistemas de produção, e mesmo com a crescente adoção dessas tecnologias por parte dos produtores, a margem da atividade segue pressionada.

Os sistemas de recria-engorda de bovinos de corte, de forma geral, são aportados com maior nível tecnológico que os sistemas de cria. Em função do até então intransponível período gestacional da vaca de cria, a recria-engorda apresenta maior flexibilidade da produção, com melhor resposta à intensificação no curto e médio prazo. Nesses sistemas, após as despesas com a aquisição dos animais, a nutrição é o componente mais significativo na formação dos custos de produção (Kumar et al., 2012). Nesse sentido, os sistemas conduzidos exclusivamente a pasto tendem a ser mais competitivos, pois apresentam despesas alimentares inferiores (Pereiraet al., 2018). Além disso, o olhar social sobre a sustentabilidade da produção é apontado como o principal fator a influenciar a pecuária moderna (Carvalho, 2005). Dessa forma, afora o aspecto econômico, a intensificação a pasto é tida como a alternativa de exploração pecuária mais relevante, também, do ponto de vista sócioambiental (Barcellos et al., 2008).

O uso massivo de insumos, per se, tem sido tratado como sinônimo de intensificação na pecuária de corte. Na verdade, intensificação pode ser definida como a obtenção do maior rendimento possível por unidade de área disponível (Moraes et al., 2013). A otimização no uso dos recursos, pela simples adoção de processos produtivos mais eficientes, pode 
repercutir em um processo de intensificação de baixo custo, demandando, basicamente, a aplicação de conhecimentos sobre o sistema de produção. Por outro lado, o uso desmedido de insumos pode gerar, em determinadas situações, custos superiores aos ganhos produtivos. Além disso, aspectos financeiros devem ser levados em conta para evitar descapitalização, prejuízos ao fluxo de caixa e/ou o endividamento. Entende-se que a intensificação dos sistemas produtivos deve ser evolutiva, passando pela adequação e domínio dos processos até a aplicação consciente de tecnologias de insumos, indo desde a escolha da categoria animal a ser utilizada sobre determinada base forrageira a própria decisão de implantação de uma dada espécie forrageira.

A capacidade decisória do gestor para avaliar e discernir situações e momentos dentro do sistema produtivo é fator basilar na viabilidade da recreia-engorda de bovinos de corte. A possibilidade de simular cenários, a partir de dados reais, que levem em consideração os fatores mutáveis dentro de um sistema de produção pode contribuir para o processo de tomada de decisão. O presente estudo tem por objetivo avaliar o desempenho bioeconômico de sistemas a pasto de recria-engorda de bovinos de corte, diferindo no nível de intensificação da base alimentar (módulos de manejo), categoria animal e nos arranjos comerciais (preços de compra e venda dos animais).

\section{Metodologia}

O presente estudo utilizou-se de um método quali-quantitativo no qual, através de dados reais de campo, foram criados cenários para a avaliação de resultados econômicos e produtivos, a partir de arranjos entre módulos de manejo, categorias de animais e conjuntura de preços de compra e venda (Pereira et al., 2018).

Foram simulados, a partir de dados de uma propriedade localizada na região da Campanha do Rio Grande do Sul (RS / Brasil), 27 cenários para sistemas a pasto de recria-engorda de bovinos de corte. Os cenários foram construídos em um arranjo $3 \times 3 \times 3$, constituídos por três níveis de intensificação (módulos de manejo), três categorias animais e três arranjos comerciais (preços de compra e venda dos animais). Além dos dados oriundos dos fluxos da propriedade, que trabalha com um sistema de recria-engorda de bovinos de corte em integração lavoura-pecuária (ILP) com soja, entre o período de maio de 2016 a dezembro de 2017, foram utilizados dados de referências literárias.

Os cenários foram simulados a partir da adaptação de planilhas eletrônicas do Microsoft Excel® descritas por Canellas (2014). Este sistema está estruturado em um modelo dividido em três módulos conversacionais para um horizonte de cinco anos: i) Sistemas de Alimentação; ii) Desempenho Animal e iii) Resumo e Análise Econômica. As alterações nas planilhas permitiram a aplicação de um maior número de preços de venda em diferentes categorias, para a simulação de diferentes cenários comerciais. Além disso, a construção de planilhas anexas possibilitou análises e comparações adicionais entre as variáveis estudadas, conferindo uma melhor visualização e interpretação dos resultados de 81 cenários detalhados por Silva (2019). Os indicadores utilizados para a avaliação dos cenários foram o tempo de fazenda (TF), o ganho de peso (GP), o ganho médio diário (GMD), a margem por cabeça/ano e a margem por hectare (ha)/ano. As despesas consideradas foram as que englobam o custo operacional efetivo (COE), como mão de obra, sanidade, nutrição e aquisição de animais.

Os recursos alimentares utilizados na recria-engorda dos animais na propriedade, denominados como módulos de manejo, são o campo nativo (CN) e a pastagem anual de inverno (PI) em sucessão à soja. Também foi considerado um recurso alimentar adicional com a simulação da implantação de pastagem anual de verão (PV) no sistema. Para isso, foi utilizado o case da propriedade, que já trabalhou com este tipo de base forrageira, e referências literárias da região. Na construção dos cenários foram utilizados três sistemas com diferentes níveis de intensificação, podendo ser considerado baixo o sistema constituído pelo módulo de manejo $\mathrm{CN}$, intermediário o sistema constituído pelo módulo de manejo $\mathrm{CN}+\mathrm{PI}$ e alto o sistema constituído pelo módulo de manejo CN + PI + PV. 
O período de uso das pastagens cultivadas e o GMD, baseados no histórico da propriedade, foram de julho a setembro para PI (90 dias), com GMD de 0,850 kg/dia em julho e agosto e de 0,950 kg/dia em setembro, e de dezembro a março para PV (120 dias), com GMD de 0,600 kg/dia em dezembro e março e de 0,750 kg/dia em janeiro e fevereiro. O GMD do CN foi ajustado a cada três meses, conforme a estação do ano. Na primavera o GMD foi de $0,480 \mathrm{~kg} / \mathrm{dia}$, no verão de $0,373 \mathrm{~kg} / \mathrm{dia}$, no outono de $0,215 \mathrm{~kg} / \mathrm{dia}$ e no inverno de $0,053 \mathrm{~kg} / \mathrm{dia}$. Os custos com alimentação foram de R \$60,00/ha para PI e R \$ 700,00/ha para PV. Estes custos foram anuais, correspondentes ao período de uso das respectivas pastagens. No CN não foram computados custos.

A PI é de baixo custo, pois apresenta ressemeadura natural de azevém (Loliummultiflorum) e se vale dos recursos empregados na cultura da soja, sendo utilizada apenas adubação nitrogenada (uréia), sem quantidade específica, em áreas que, por avaliação visual, julga-se necessário. Entretanto, o tempo de uso e o GMD podem ser considerados de moderados a baixos. Além disso, a capacidade de suporte (CS) definida para esta pastagem foi de 1,11 unidade animal (UA - $450 \mathrm{~kg}$ de peso corporal), atingindo uma carga animal (CA) média de apenas 0,95 UA, valores também baixos para este recurso alimentar. A PV apresentou GMD aquém do potencial e do verificado na literatura, pelo baixo índice pluviométrico característico da região no período de verão. Também por isso a CA ficou em apenas 1,79 UA, apesar da CS definida de 2,11 UA. A CS média/ano definida no CN foi de 0,82 UA, sendo atingida uma CA média de 0,79 UA.

Foram identificadas três principais categorias animais comercializadas na propriedade: i) bezerro desmamado (6 a 8 meses de idade); ii) novilho de ano (12 a 14 meses de idade); iii) vaca de descarte (sem idade definida). As épocas predominantes de aquisição destes animais são maio para o bezerro e a vaca e novembro para o novilho. Os meses de entrada dos animais no sistema foram sempre respeitados na simulação, independente do mês de maior ou menor saldo entre CS e CA, não sendo os meses de entrada alterados para o melhor ajuste da CA, buscando-se manter a simulação o mais fiel possível a realidade da propriedade. A saída dos animais do sistema ocorreu quando estes atingiram o peso final (PF) alvo, definido com base em médias da propriedade, assim como o peso inicial. Os pesos iniciais e finais médios foram, respectivamente, 172,33 e 497,00 kg para o bezerro, 243,00 e 497,00 kg para o novilho e de 366,00 e 494,00 kg para a vaca.

Os preços de comercialização também foram determinados a partir de valores médios praticados na propriedade. Na formação dos arranjos comerciais foram utilizados preços de compra e venda, respectivamente, alto/baixo (desfavorável), médio/médio (médio) e baixo/alto (favorável). Como critério de variação para a definição dos preços alto e baixo foi utilizado o valor de oscilação de 10,0\% do preço médio (Tabela 1).

Tabela 1. Arranjos comerciais e variação no valor de transferência de acordo com a categoria animal.

\begin{tabular}{llccc}
\hline \multirow{2}{*}{ Categoria animal } & \multirow{2}{*}{ Arranjo comercial } & \multicolumn{2}{c}{ Preço (R\$/kg) } & Variação (\%) \\
\cline { 3 - 5 } Bezerro & Desfavorável & Compra & Venda & $-25,3 \%$ \\
& Médio & 6,41 & 4,79 & $-8,7 \%$ \\
& Favorável & 5,83 & 5,32 & $11,4 \%$ \\
\cline { 2 - 5 } Novilho & Desfavorável & 5,25 & 5,85 & $-12,9 \%$ \\
& Médio & 5,50 & 4,79 & $6,4 \%$ \\
Vaca & Favorável & 5,00 & 5,32 & $30,0 \%$ \\
\cline { 2 - 5 } & Desfavorável & 4,50 & 5,85 & $-17,5 \%$ \\
& Médio & 4,74 & 3,91 & $0,7 \%$ \\
\hline
\end{tabular}


Os preços de venda ainda sofreram ajustes conforme o mês de comercialização dos animais. Os ajustes foram realizados com base na oscilação mensal média do estado do RS em relação ao preço médio anual (Figura 1).

Figura 1. Variação mensal do preço médio anual do boi gordo ( $\mathrm{R} \$ \mathrm{~kg}$ vivo).

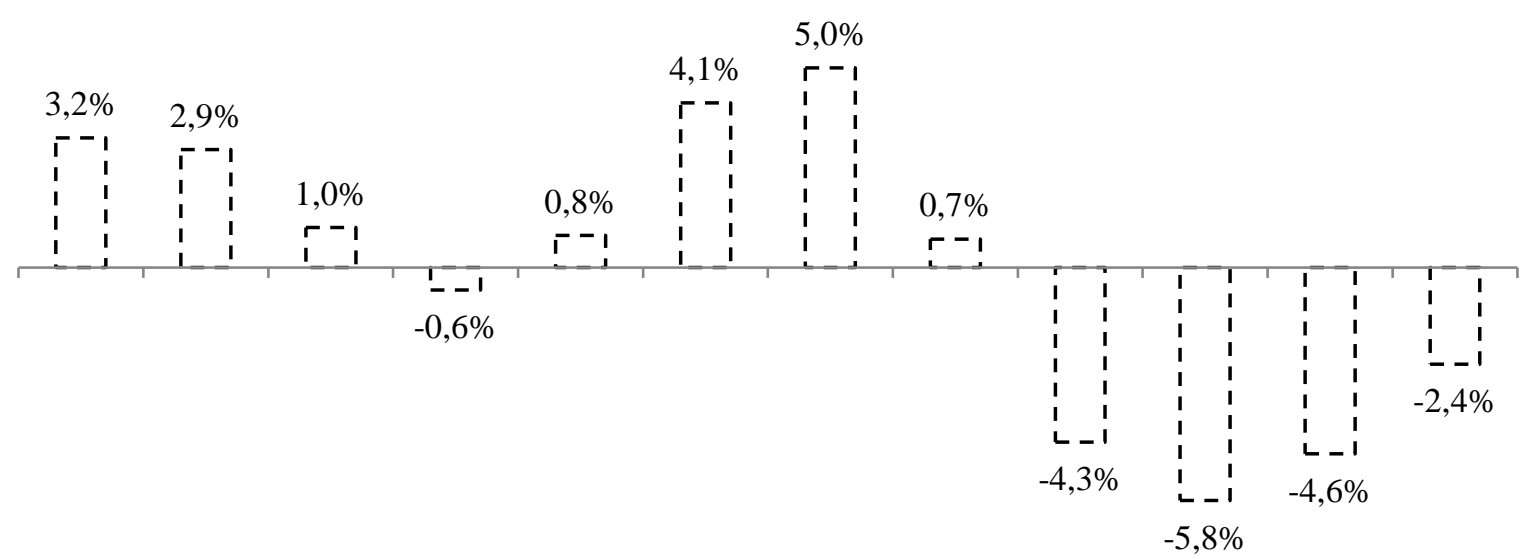

jan fev mar abr mai jun jul ago set out nov dez

Fonte: os autores, através de dados obtidos do Informativo Nespro \& Embrapa (2015, 2016 e 2018).

Com as variáveis estabelecidas, o conjunto de cenários (Tabela 2) foi modelado e as simulações foram realizadas.

Tabela 2. Conjunto de cenários avaliados.

\begin{tabular}{|c|c|c|c|}
\hline Arranjo comercial & Categoria animal & Módulo de manejo & Cenário \\
\hline \multirow{9}{*}{ Desfavorável } & \multirow{3}{*}{ Bezerro } & $\mathrm{CN}$ & 1 \\
\hline & & $\mathrm{CN}+\mathrm{PI}$ & 2 \\
\hline & & $\mathrm{CN}+\mathrm{PI}+\mathrm{PV}$ & 3 \\
\hline & \multirow{3}{*}{ Novilho } & $\mathrm{CN}$ & 4 \\
\hline & & $\mathrm{CN}+\mathrm{PI}$ & 5 \\
\hline & & $\mathrm{CN}+\mathrm{PI}+\mathrm{PV}$ & 6 \\
\hline & \multirow{3}{*}{ Vaca } & $\mathrm{CN}$ & 7 \\
\hline & & $\mathrm{CN}+\mathrm{PI}$ & 8 \\
\hline & & $\mathrm{CN}+\mathrm{PI}+\mathrm{PV}$ & 9 \\
\hline \multirow{9}{*}{ Médio } & \multirow{3}{*}{ Bezerro } & $\mathrm{CN}$ & 10 \\
\hline & & $\mathrm{CN}+\mathrm{PI}$ & 11 \\
\hline & & $\mathrm{CN}+\mathrm{PI}+\mathrm{PV}$ & 12 \\
\hline & \multirow{3}{*}{ Novilho } & $\mathrm{CN}$ & 13 \\
\hline & & $\mathrm{CN}+\mathrm{PI}$ & 14 \\
\hline & & $\mathrm{CN}+\mathrm{PI}+\mathrm{PV}$ & 15 \\
\hline & \multirow{3}{*}{ Vaca } & $\mathrm{CN}$ & 16 \\
\hline & & $\mathrm{CN}+\mathrm{PI}$ & 17 \\
\hline & & $\mathrm{CN}+\mathrm{PI}+\mathrm{PV}$ & 18 \\
\hline \multirow{9}{*}{ Favorável } & \multirow{3}{*}{ Bezerro } & $\mathrm{CN}$ & 19 \\
\hline & & $\mathrm{CN}+\mathrm{PI}$ & 20 \\
\hline & & $\mathrm{CN}+\mathrm{PI}+\mathrm{PV}$ & 21 \\
\hline & \multirow{3}{*}{ Novilho } & $\mathrm{CN}$ & 22 \\
\hline & & $\mathrm{CN}+\mathrm{PI}$ & 23 \\
\hline & & $\mathrm{CN}+\mathrm{PI}+\mathrm{PV}$ & 24 \\
\hline & \multirow{3}{*}{ Vaca } & $\mathrm{CN}$ & 25 \\
\hline & & $\mathrm{CN}+\mathrm{PI}$ & 26 \\
\hline & & $\mathrm{CN}+\mathrm{PI}+\mathrm{PV}$ & 27 \\
\hline
\end{tabular}




\section{Resultados e Discussão}

O bezerro, mesmo em uma conjuntura comercial desfavorável, apresenta margens por ha/ano positivas e com respostas crescentes à intensificação do sistema com a utilização de PI e PV em relação aos módulos de manejo CN e CN+PI, apesar deste comportamento não ser verificado na curva de margem por cabeça/ano (Figura 2).

Figura 2. Bezerro em arranjo comercial desfavorável - Margem por cabeça/ano e margem por ha/ano nos diferentes módulos de manejo $(\mathrm{R} \$)$.

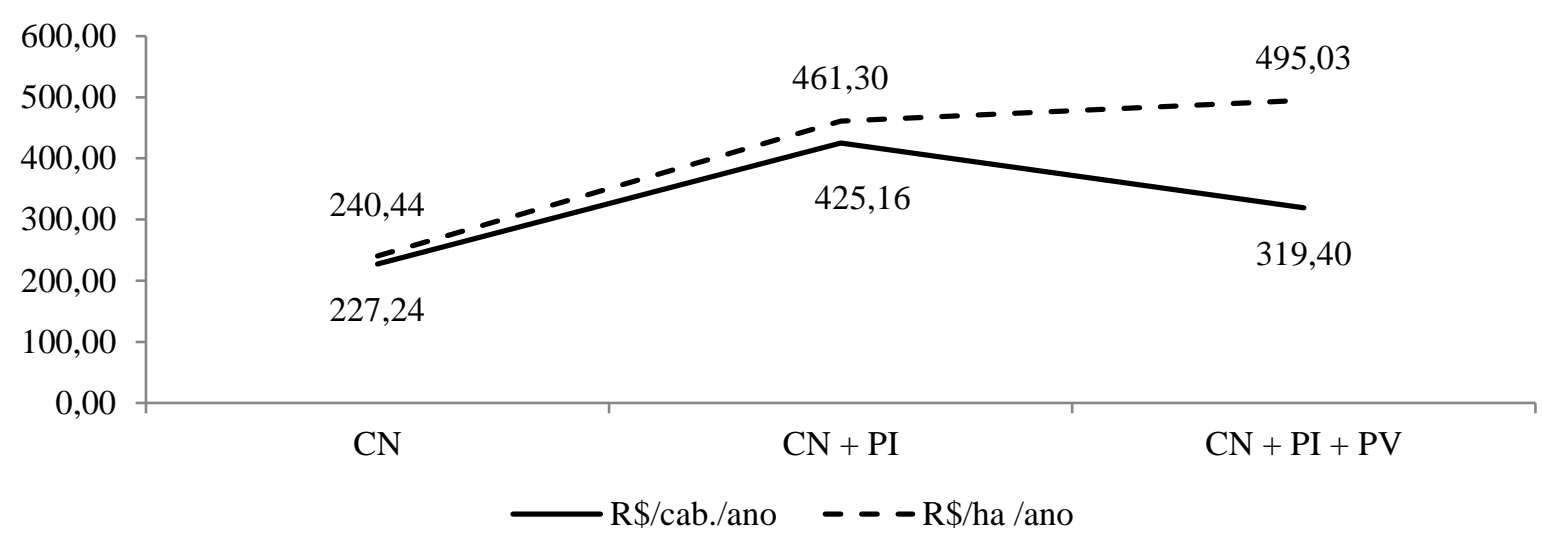

Fonte: Autores.

A margem por cabeça/ano decresce quando há a intensificação do sistema pela implantação da PV em relação ao módulo de manejo CN + PI, pois há um considerável aumento no custo por cabeça. Quando avaliado o ganho por área, pela margem por ha/ano, observa-se ganho no sistema pela maior lotação proporcionada pela PV. Contudo, através do comportamento da curva da margem/ha/ano, verifica-se que o ganho por área obtido com a intensificação com PI em relação ao módulo de manejo $\mathrm{CN}$ é superior ao obtido pela intensificação com PV em relação ao módulo de manejo $\mathrm{CN}+\mathrm{PI}$.

O comportamento crescente da margem por ha/ano verificado na categoria bezerro acompanhando o aumento do nível de intensificação não foi observado nas categorias novilho (Figura 3) e vaca (Figura 4), decrescendo tanto da margem por cabeça/ano quanto na margem por ha/ano com a intensificação do sistema pela implantação da PV em relação ao módulo de manejo CN + PI. 
Figura 3. Novilho em arranjo comercial desfavorável - Margem por cabeça/ano e margem por ha/ano nos diferentes módulos de manejo $(\mathrm{R} \$)$.

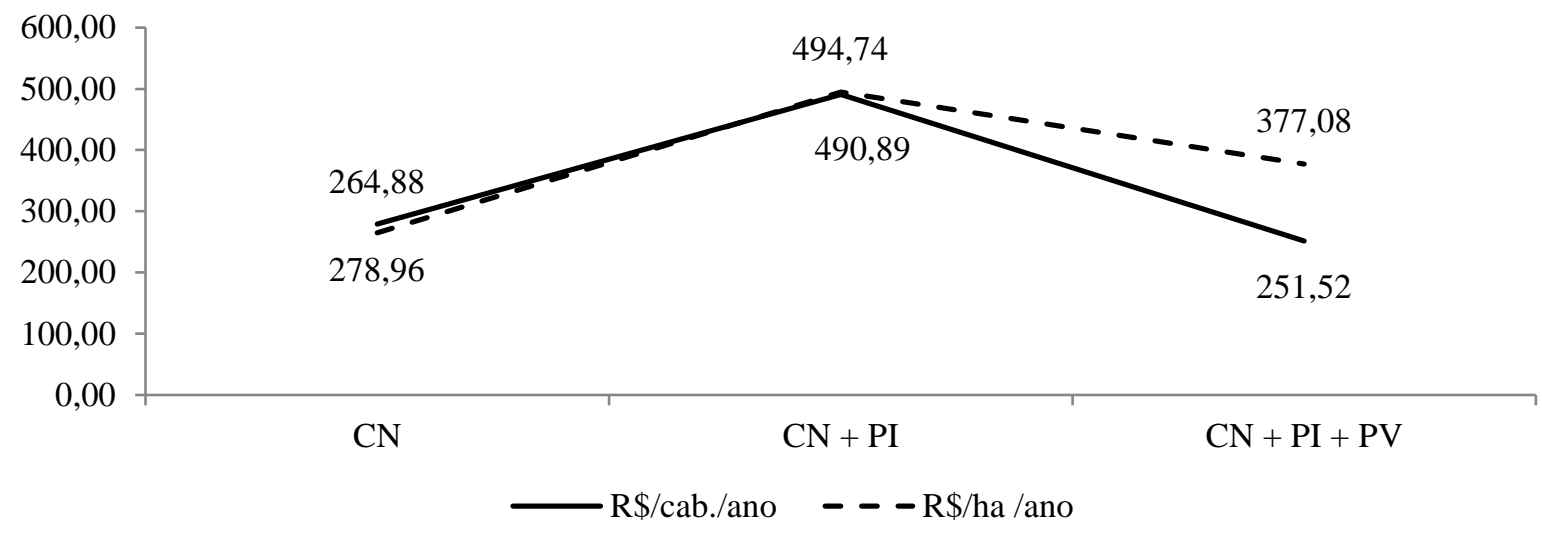

Fonte: Autores.

Figura 4. Vaca em arranjo comercial desfavorável - Margem por cabeça/ano e margem por ha/ano nos diferentes módulos de manejo $(\mathrm{R} \$)$.

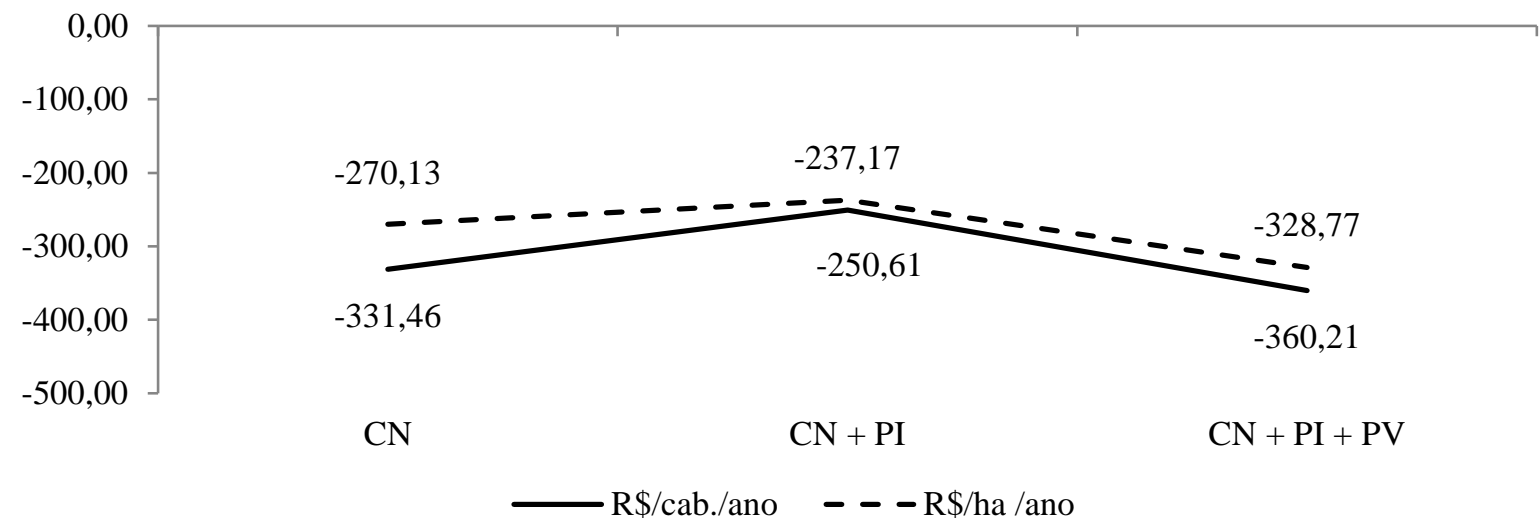

Fonte: Autores.

Com o maior custo por cabeça na aquisição de categorias mais pesadas e, consequentemente, menor número de cabeças por área para atingir a CA, mesmo com maior CS proporcionada pela PV, em contexto comercial desfavorável verificou-se decréscimo das margens nos sistemas mais intensificados do novilho e da vaca. Nesta conjuntura comercial todas as categorias sofreram um deságio entre o valor de venda e compraem $\mathrm{R} \$ / \mathrm{kg}$ (Tabela 1). Apesar do deságio do bezerro ser superior ao do novilho (25,3 vs $12,9 \%)$ e ao da vaca (25,3 vs 17,5\%), nestas categorias, além do maior peso médio de compra, que gera um prejuízo maior pela maior quantidade de quilogramas que sofrem a perda de valor, o GP e o TF no sistema são inferiores aos do bezerro, fatores que colaboraram para o maior prejuízo comercial. Ao passo que as categorias mais pesadas, nesta conjuntura, foram prejudicadas pela menor agregação de peso no sistema e maior concentração do prejuízo comercial pelo menor período de permanência, o bezerro foi beneficiado por estes mesmos aspectos (Tabela 3). 
Tabela 3. Tempo de fazenda (TF), ganho de peso (GP) e ganho médio diário (GMD) por categoria e módulo de manejo.

\begin{tabular}{llccc}
\hline \multicolumn{1}{c}{ Categoria animal } & Módulo de manejo & TF (meses) & GP $(\mathbf{k g})$ & GMD $(\mathbf{k g} / \mathbf{d i a})$ \\
\hline \multirow{3}{*}{ Bezerro } & CN & 40 & 334,15 & 0,278 \\
& CN + PI & 25 & 341,99 & 0,469 \\
& CN + PI + PV & 20 & 338,33 & 0,559 \\
\cline { 2 - 5 } Novilho & CN & 30 & 259,93 & 0,291 \\
& CN + PI & 16 & 265,61 & 0,440 \\
& CN + PI + PV & 15 & 253,92 & 0,563 \\
\cline { 2 - 5 } Vaca & CN & 18 & 136,11 & 0,254 \\
& CN + PI & 10 & 141,94 & 0,499 \\
& CN + PI + PV & 9 & 143,57 & 0,548 \\
\hline
\end{tabular}

Fonte: Autores.

O menor TF, que muitas vezes, por si só, é tratado como sinônimo de eficiência produtiva e perseguido de forma indiscriminada, foi determinante para o decréscimo da margem do sistema com nível alto de intensificação (CN + PI + PV) em relação ao intermediário $(\mathrm{CN}+\mathrm{PI})$ nas categorias mais pesadas, em um contexto comercial desfavorável. Quando a conjuntura comercial se encontra em patamares médios, o bezerro continua apresentando ganhos crescentes conforme o sistema é intensificado pela incorporação de pastagens cultivadas nos módulos de manejo (Figura 5). Entretanto, verificou-se que o crescimento da margem por ha/ano com a intensificação pela implantação da PV em relação ao módulo de manejo CN + PI é superior ao obtido na conjuntura comercial desfavorável, ao passo que ocorre o inverso na intensificação com a PI em relação ao módulo CN. Enquanto na conjuntura comercial desfavorável o acréscimo da margem por ha/ano entre os módulos $\mathrm{CN}$ e $\mathrm{CN}$ + PI foi de 91,9\% e entre os módulos CN + PI e CN + PI + PV foi de 7,3\%, na conjuntura comercial média estes acréscimos foram de, respectivamente, 87,1 e $25,7 \%$. Neste contexto, o deságio do bezerro é de $8,7 \%$, enquanto o novilho e a vaca são beneficiados com um pequeno ágio entre os valores médios de transferência ( 6,4 e $0,7 \%$, respectivamente).

Figura 5. Bezerro em arranjo comercial médio - Margem por cabeça/ano e margem por ha/ano nos diferentes módulos de manejo (R\$).

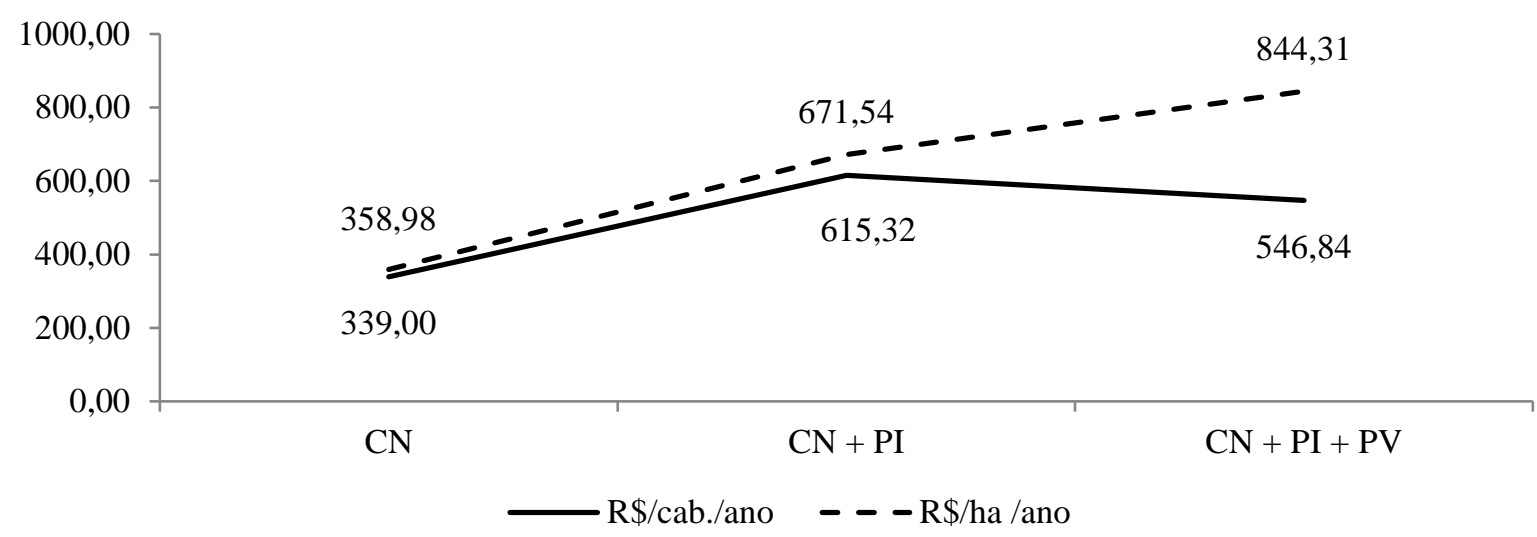

Fonte: Autores. 
Nesta conjuntura comercial o novilho passa a apresentar margens por ha/ano crescentes conforme o sistema é intensificado, assim como o bezerro (Figura 6). Os comportamentos das curvas das margens por ha/ano do novilho são similares ao do bezerro em relação ao contexto comercial. A categoria, em relação ao arranjo de preços desfavorável, mostra um decréscimo no aumento da margem com a intensificação pela PI em relação ao módulo de manejo CN, que passa de 86,8\% no contexto de preços desfavorável para $79,1 \%$ no contexto de preços médio. Já com a intensificação pela PV em relação ao módulo de manejo CN + PI a margem passa de um decréscimo de $23,8 \%$ no contexto de preços desfavorável para um acréscimo de $14,7 \%$ com os preços médios.

Figura 6. Novilho em arranjo comercial médio - Margem por cabeça/ano e margem por ha/ano nos diferentes módulos de manejo (R\$).

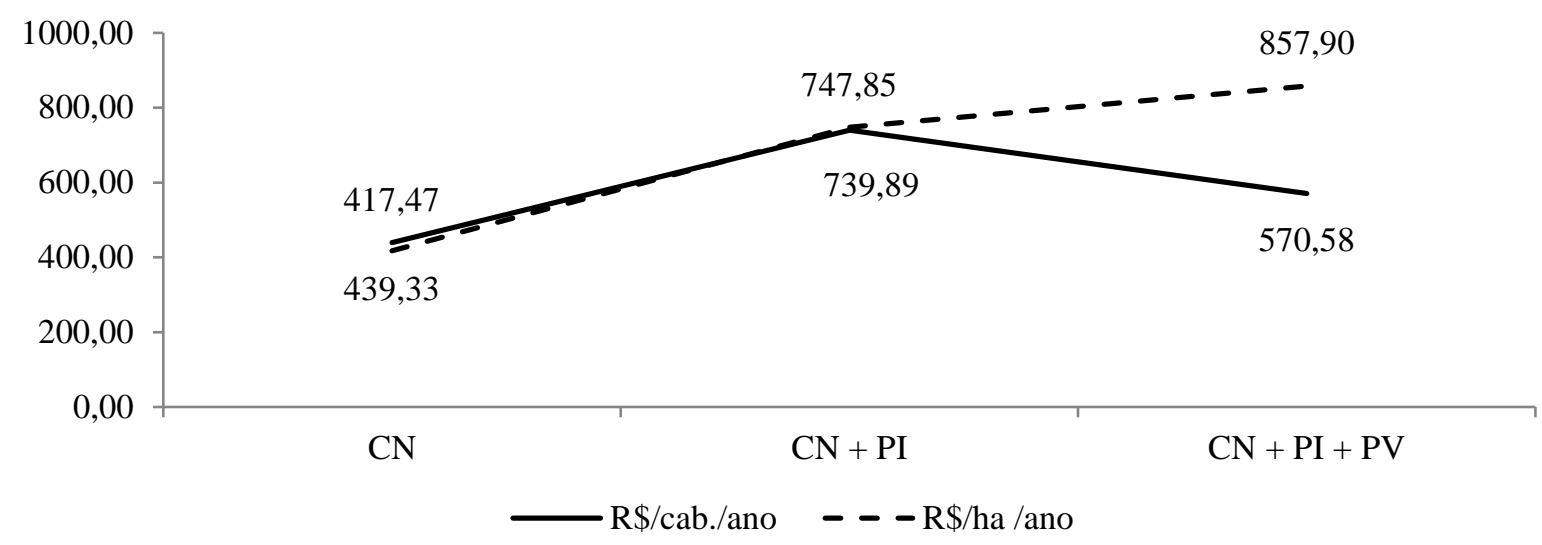

Fonte: Autores.

Mesmo com um cenário de preços médios a vaca permanece com decréscimo da margem por ha/ano quando há a implantação da PV em relação ao módulo de manejo CN + PI. Contudo, a categoria passa a apresentar valores positivos em todos os módulos de manejo (Figura 7).

Figura 7. Vaca em arranjo comercial médio - Margem por cabeça/ano e margem por ha/ano nos diferentes módulos de manejo $(\mathrm{R} \$)$.

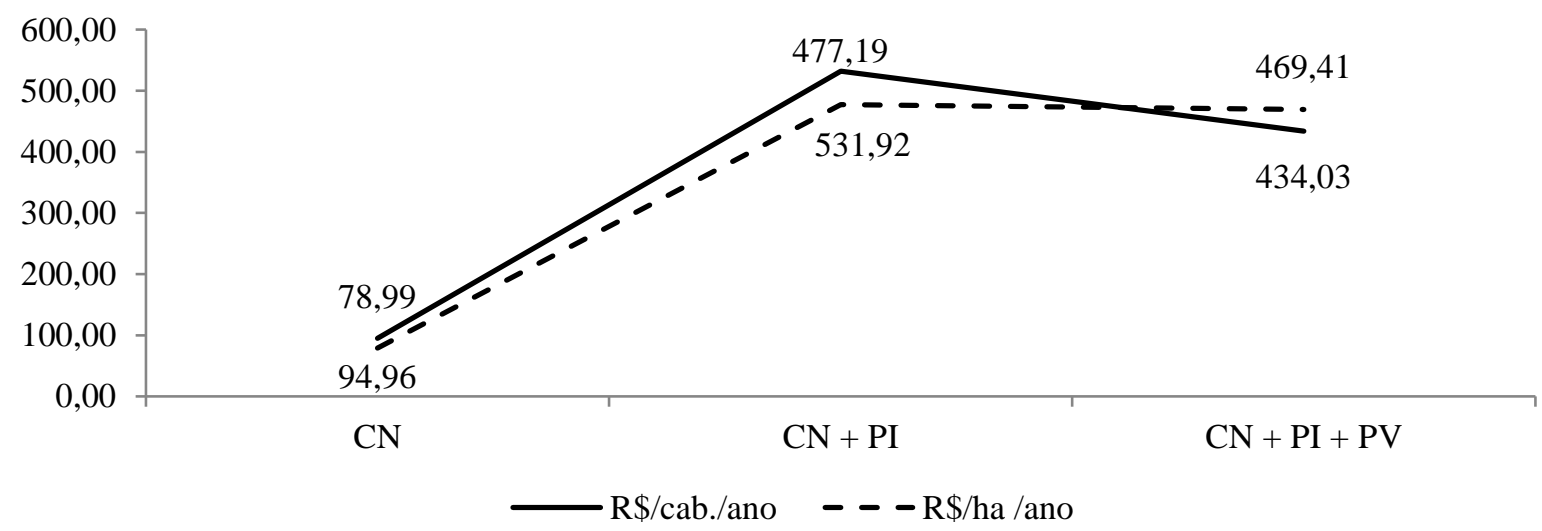

Fonte: Autores. 
Em uma conjuntura de preços favoráveis todas as categorias se beneficiam com ágio no valor de venda em relação ao de compra, correspondentes a 11,4\% para o bezerro, 30,0\% para o novilho e 22,9\% para a vaca. O bezerro apresenta a menor melhora entre o módulo $\mathrm{CN}$ e $\mathrm{CN}+\mathrm{PI}(85,6 \%)$ e a maior entre $\mathrm{CN}+\mathrm{PI}$ e CN + PI + PV (35,5\%) entre todas as conjunturas comerciais apresentadas, mantendo o comportamento de melhorar proporcionalmente mais o sistema mais intensivo e de maior desembolso por cabeça conforme o cenário de preços melhora (Figura 8).

Figura 8. Bezerro em arranjo comercial favorável - Margem por cabeça/ano e margem por ha/ano nos diferentes módulos de manejo $(\mathrm{R} \$)$.

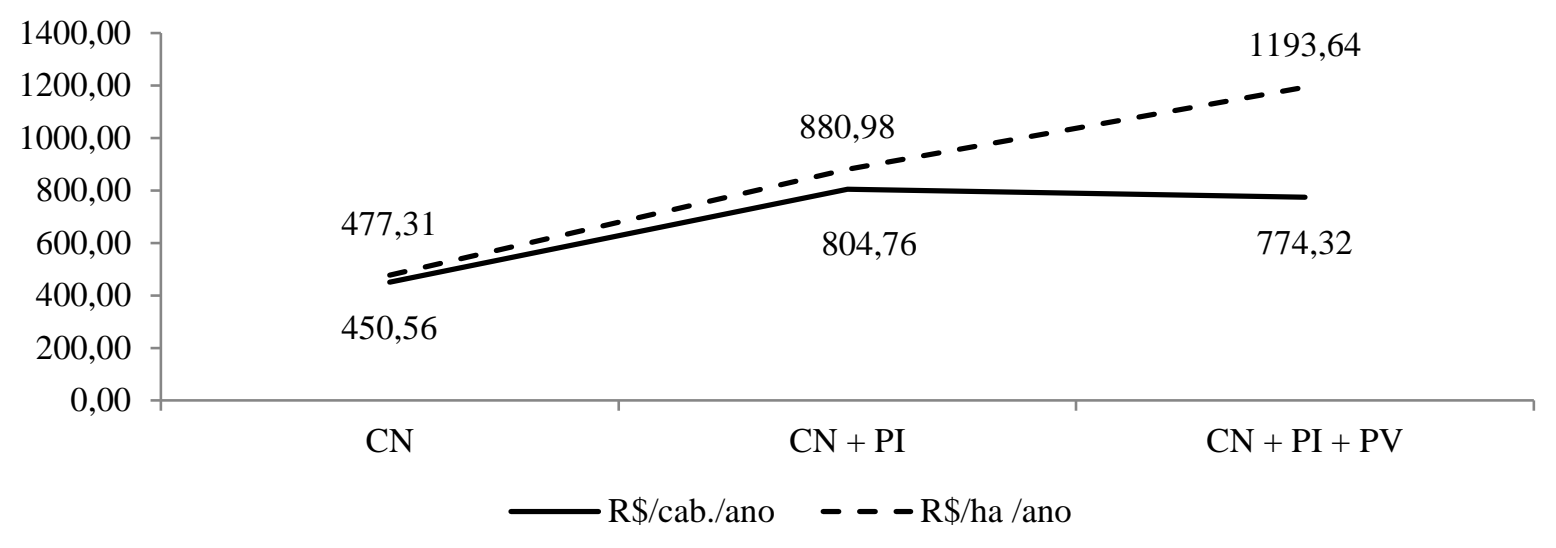

Fonte: Autores.

O novilho mantém o mesmo padrão de comportamento entre os níveis de intensificação que o bezerro, melhorando a margem por ha/ano em 75,6\% entre $\mathrm{CN}$ e CN + PI e 35,5\% entre CN + PI e CN + PI + PV (Figura 9).

Figura 9. Novilho em arranjo comercial favorável - Margem por cabeça/ano e margem por ha/ano nos diferentes módulos de manejo $(\mathrm{R} \$)$.

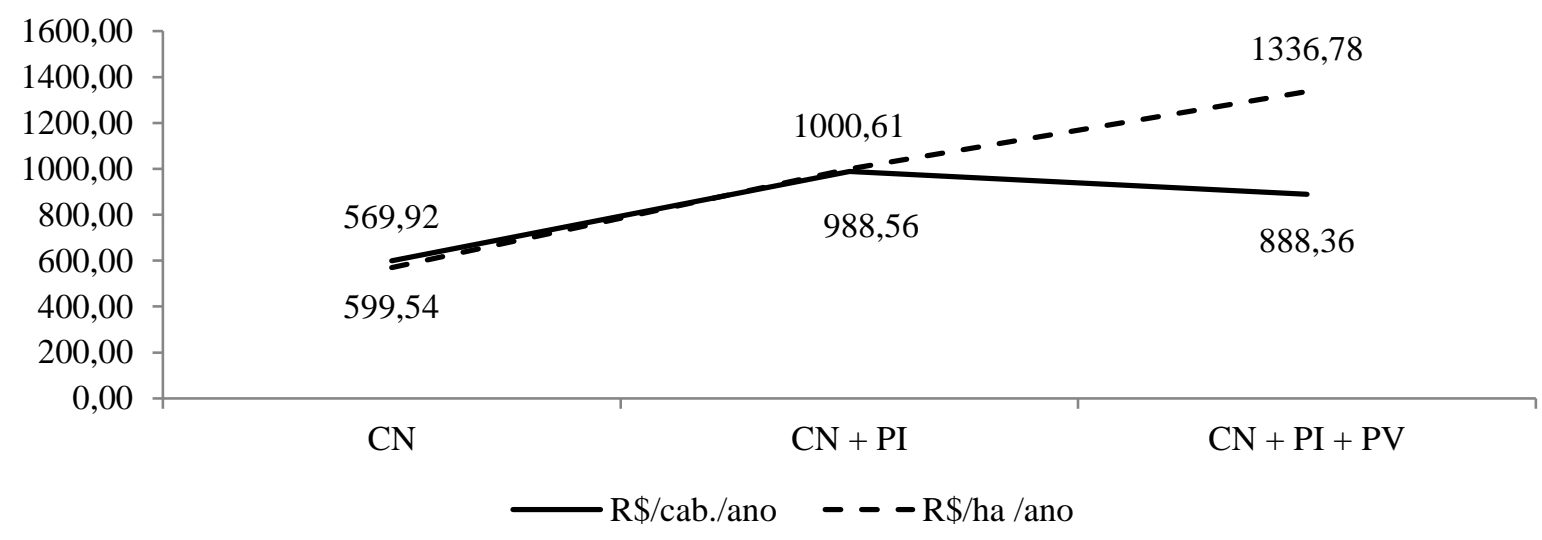

Fonte: Autores.

A vaca obtém uma melhora crescente com o processo de intensificação do sistema apenas na conjuntura comercial favorável (Figura 10). Entretanto, ainda assim, essa melhora é inferior a obtida nas demais categorias. 
Figura 10. Vaca em arranjo comercial favorável - Margem por cabeça/ano e margem por ha/ano nos diferentes módulos de manejo $(\mathrm{R} \$)$.

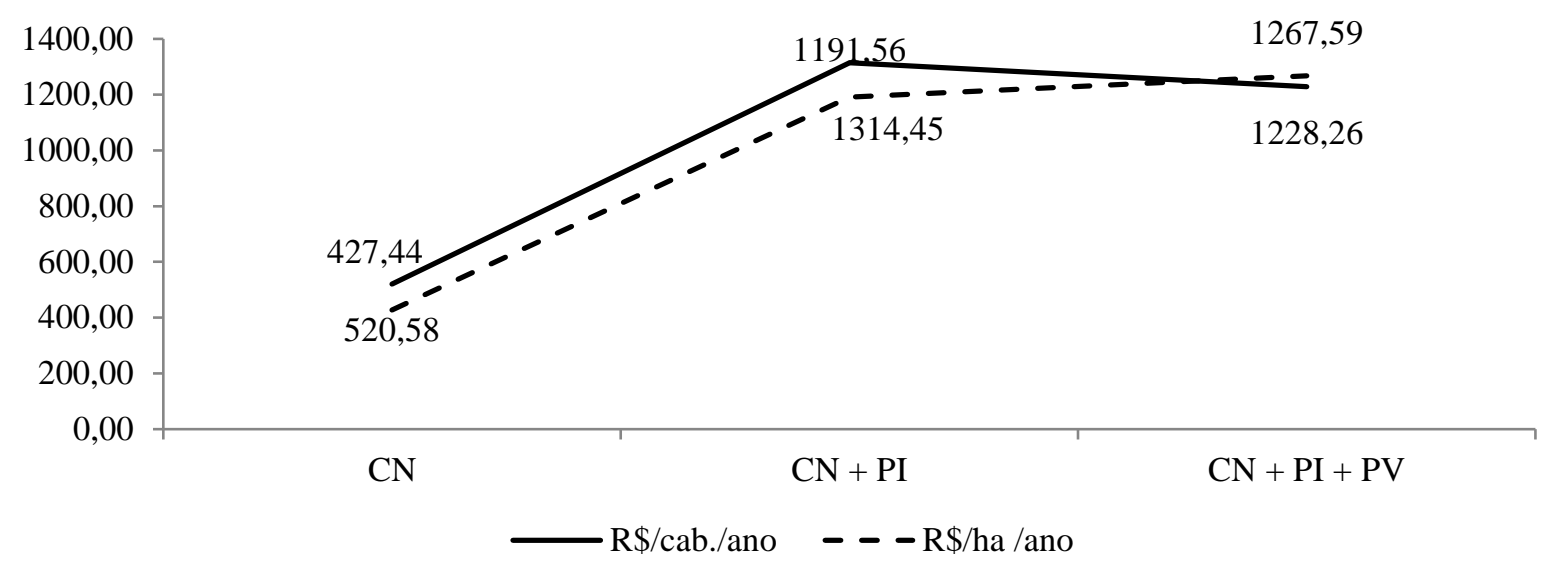

Fonte: Autores.

Com exceção da vaca, que melhora proporcionalmente mais com a intensificação moderada pela implantação da PI em relação ao módulo de manejo CN que pela implantação da PV em relação ao módulo CN + PI conforme a conjuntura comercial melhora, as demais categorias tendem a diminuir a proporção do crescimento da margem por ha/ano de CN para CN + PI e aumentá-la de CN + PI para CN + PI + PV. Esse resultado indica que sistemas com maior investimento em recursos forrageiros respondem melhor à melhora da conjuntura comercial que sistemas com menor investimento em recursos forrageiros. Isso, em nível de campo, pode sinalizar aos gestores de sistemas mais intensivos a exigência de uma maior atenção comercial na compra e venda de animais, principalmente em momentos comercialmente desfavoráveis, sendo estes negócios de maior risco que sistemas menos intensivos.

As categorias de maior peso na aquisição tendem a apresentar perda de competitividade em cenários de preços desfavoráveis, pois apresentam maior quantidade de quilogramas adquiridos em contexto desfavorável, menor TF para diluir este prejuízo e menor GP no sistema para incremento da receita. O bezerro, apesar de ser a categoria mais impactada negativamente pelo valor de transferência entre compra e venda, apresenta maior TF e diluí mais eficientemente os prejuízos comerciais. Além disso, o maior GP confere uma maior agregação de valor ao sistema. Dessa forma, mesmo com o aumento do desembolso por cabeça com a intensificação do sistema e em conjuntura comercial desfavorável, o bezerro demonstra desempenho financeiro crescente por área. Já quando a conjuntura comercial passa a melhorar, indo para patamares médios, o novilho tende a apresentar melhor resposta, já sem sofrer deságio no preço de venda. Entretanto, em um contexto de preços favoráveis, apesar do maior ágio apresentado pelo novilho, em um contexto de intensificação moderada, como é o módulo de manejo CN + PI com PI de baixo custo em sucessão à soja, a vaca apresentou a melhor margem por área. Apesar do menor GP da categoria no sistema, os ganhos comerciais pelo ágio da venda em uma grande quantidade de quilogramas adquiridos, somados ao menor TF, garantem à vaca o melhor desempenho. Contudo, se deve ter atenção que muito deste ganho é financeiro, não produtivo.

Quando se avalia a margem por cabeça/ano, em qualquer arranjo de preços e categoria animal, o módulo de manejo $\mathrm{CN}+\mathrm{PI}+\mathrm{PV}$ perde competitividade em relação ao módulo CN + PI, inclusive, em alguns casos, em relação ao CN. Isso ocorre pelo maior desembolso por cabeça/ano gerado pela incorporação da PV. Entretanto, quando avaliada a margem por ha/ano, considerando a maior CS e CA, em apenas $11,1 \%$ dos cenários (3/27) ocorreu perda de margem com a intensificação do sistema pela incorporação da PV. Com isso, salienta-se para a importância de o que e como se deseja avaliar para aplicar o indicador mais adequado à análise. Destaca-se, também, que os resultados e suas interpretações podem ser fortemente alterados 
caso variáveis como o GMD e os custos, principalmente das pastagens hibernais, que neste caso são de menor custo em um sistema de ILP de baixo uso de insumos, sejam alterados. Para a correta análise dos resultados, alerta-se, ainda, para os custos aqui computados. Deve-se ter em conta que os custos considerados foram apenas os desembolsados relacionados ao COE, como mão de obra, sanidade e nutrição, além de aquisição dos animais. Outro ponto que impactou significativamente nos resultados foi o ganho financeiro por aspectos comerciais do valor de transferência entre preços de compra e venda. Consideraram-se os extremos nos valores favoráveis (baixo/alto) e desfavoráveis (alto/baixo).

Ao analisar os resultados de margem por ha/ano com o auxílio de uma escala de cores (Tabela 4), em que tons vermelhos mais intensos representam valores inferiores e tons verdes mais intensos valores superiores, sob uma ótica do contexto comercial, não mais da categoria animal como nos gráficos anteriores, observa-se que o módulo de manejo com nível alto de intensificação $(\mathrm{CN}+\mathrm{PI}+\mathrm{PV})$ apresenta margens superiores em todos os contextos comerciais, apenas variando a categoria animal.

Tabela 4. Margem por ha/ano (R\$) de cada categoria animal e módulo de manejo nas diferentes conjunturas comerciais ${ }^{1}$.

\begin{tabular}{ccccc}
\hline \multirow{2}{*}{ Conjuntura comercial } & Categoria animal & \multicolumn{3}{c}{ Módulo de manejo } \\
\cline { 3 - 5 } & & CN & CN + PI & CN + PI + PV \\
\hline \multirow{2}{*}{ Desfavorável } & Bezerro & 240,44 & 461,30 & 495,03 \\
& Novilho & 264,88 & 494,74 & 377,08 \\
\hline \multirow{2}{*}{ Médio } & Vaca & $-270,13$ & $-237,17$ & $-328,77$ \\
& Bezerro & 358,98 & 671,54 & 844,31 \\
\hline \multirow{2}{*}{ Favorável } & Novilho & 417,47 & 747,85 & 469,41 \\
\hline & Vaca & 78,99 & 477,19 & 1193,64 \\
\hline
\end{tabular}

${ }^{1}$ Escala de cores do menor valor (vermelho) ao maior valor (verde) correspondentes a conjuntura comercial.

Fonte: Autores.

Em conjuntura comercial desfavorável, o bezerro apresenta a melhor margem entre as categorias no módulo de manejo CN + PI + PV, sendo o melhor cenário do contexto comercial desfavorável, seguido pelo novilho em CN + PI. Já a vaca, neste contexto, apresenta valores negativos em todos os módulos de manejo. Em conjuntura comercial de preços médios, o novilho apresenta as melhores margens entre as categorias em todos os módulos de manejo, sendo o melhor cenário do contexto comercial em módulo de manejo $\mathrm{CN}+\mathrm{PI}+\mathrm{PV}$, seguido pelo bezerro em mesmo módulo de manejo. Em conjuntura comercial de preços favoráveis, a melhor margem ocorre, novamente, com o novilho em módulo de manejo CN + PI + PV, seguido pela vaca em mesmo módulo.

Os valores absolutos apresentados neste trabalho não devem ser extrapolados para todos os sistemas de produção. No entanto, apesar disso, o comportamento das curvas pode ser utilizado como parâmetro, desde que em sistemas de produção com características semelhantes. Neste sentido, ao avaliar a resposta pela margem/ha/ano em sistemas de recria-engorda em ILP, com estes níveis de uso de tecnologia, pode-se inferir que: i) quando em contexto comercial desfavorável, o bezerro seja a categoria mais indicada de se trabalhar com nível mais alto de intensificação do módulo de manejo $(\mathrm{CN}+\mathrm{PI}+\mathrm{PV})$, o novilho em nível intermediário $(\mathrm{CN}+\mathrm{PI})$ e baixo $(\mathrm{CN})$, e a vaca contra-indicada; ii) quando em contexto comercial médio o novilho 
seja a categoria mais indicada de se trabalhar em todos os níveis de intensificação do módulo de manejo (CN + PI + PV, CN + PI ou CN); e iii) quando em contexto comercial favorável o novilho seja a categoria mais indicada de se trabalhar com nível de intensificação do módulo de manejo baixo (CN) e alto (CN + PI + PV), e a vaca com nível intermediário (CN + PI).

\section{Conclusões}

Ao buscar respostas para questionamentos do cotidiano dos gestores de sistemas de recria-engorda de bovinos de corte, como a categoria animal a ser utilizada em determinada conjuntura comercial e nível de intensificação (módulo de manejo / sistema de produção), em qual momento e para qual categoria intensificar o sistema com pastagens cultivadas ou qual a interação destas variáveis, o presente trabalho trouxe à luz do conhecimento resultados pontuais de determinados contextos produtivos, alicerçadas em dados e situações reais. No entanto, apesar dos valores absolutos serem únicos, o comportamento das curvas de respostas pode ser considerado como um parâmetro, mesmo que alguns valores se alterem, desde que mantidos os aspectos dos sistemas ora apresentados.

É importante salientar que o baixo nível de uso de insumos na PI impactou significativamente os resultados e deve ser levado em consideração. O desembolso com a PV pode, também, ser considerado relativamente baixo para um maior nível de insumos que podem ser utilizados em algumas situações. Entretanto, estes parâmetros respeitaram os empregados na propriedade em questão. E, também, entende-se que hoje estes sistemas ainda podem ser considerados predominantes.

A partir do completo domínio destas e outras variáveis, os gestores de sistemas de produção de recria-engorda podem partir para intensificações mais agressivas do ponto de vista de insumos e desembolsos, como um maior aporte nutricional para as forrageiras com adubações mais pesadas, buscando aumento de produtividade. Entretanto, lançar mão de tecnologias de insumos que exigem maior desembolso sem o completo controle e entendimento do sistema de produção pode acarretar perda de competitividade.

\section{Agradecimentos}

A Estância Dona Genoveva, na pessoa do Dr. Renato Kalil e família, a Empresa Brasileira de Pesquisa Agropecuária (Embrapa) Pecuária Sul e a Coordenação de Aperfeiçoamento de Pessoal de Nível Superior - Brasil (CAPES).

O presente trabalho foi realizado com apoio da Coordenação de Aperfeiçoamento de Pessoal de Nível Superior Brasil (CAPES) - Código de Financiamento 001.

\section{Referências}

Barcellos, A. O., Ramos, A. K. B., Vilela, L., \& Martha Junior, G. B. (2008). Sustentabilidade da produção animal baseada em pastagens consorciadas e no emprego de leguminosas exclusivas, na forma de banco de proteína, nos trópicos brasileiros. Revista Brasileira de Zootecnia, 37 (suplemento especial), 51-67.

Canellas, L. C. (2014). Modelagem e simulação para análise de sistemas de recria - terminação de bovinos de corte. Tese de Doutorado em Zootecnia Universidade Federal do Rio Grande do Sul.

Carvalho, P. C. F. (2005). O manejo da pastagem como gerador de ambientes pastoris adequados à produção animal. Anais do Simpósio de Manejo de Pastagens: Teoria e Prática da Produção Animal em Pastagens, 22, 7-32.

Freitas, A. P., Guterres, M. X., Lampert, V. N., Silva, A. H. S., Barcellos, J. O. J. \& Marques, P. R. (2017). Aplicação de método de seleção de variáveis em um modelo DEA na produção de bovinos de corte. ENGEVISTA, 19(4), 881-889.

Kumar, R., Lardner, H. A., McKinnon, J. J., Christensen, D. A., Damiran, D. \& Larson, K. (2012). Comparison of alternative backgrounding systems on beef calf performance, feedlot finishing performance, carcass traits, and system cost of gain. The Professional AnimalScientist.28, 541-51.

Moraes, E. H. B. K., Moraes, K. A. K., Oliveira, A. S., Hoffmann, A., Simioni, T. A., Mousquer, C. J., Paula, D. C., Socreppa, L. M., Botini, L. A. \& Alonso, M. P. (2013). Sistemas intensivos de produção de carne bovina com uso de suplementos múltiplos. AnaisdoSimpósio

Matogrossense de Bovinocultura de Corte, II, Cuiabá, MT, UFMT.

Núcleo de Estudos em Sistemas de Produção de Bovinos de Corte e Cadeia Produtiva (NESPRO) \& Empresa Brasileirade Pesquisa Agropecuária (EMBRAPA). (2015). Informativo NESPRO \& Embrapa Pecuária Sul: Bovinocultura de Corte no Rio Grande do Sul, 2(1). 
Research, Society and Development, v. 10, n. 12, e82101213908, 2021

(CC BY 4.0) | ISSN 2525-3409 | DOI: http://dx.doi.org/10.33448/rsd-v10i12.13908

Núcleo de Estudos em Sistemas de Produção de Bovinos de Corte e Cadeia Produtiva (NESPRO) \& Empresa Brasileira de Pesquisa Agropecuária (EMBRAPA). (2016). Informativo NESPRO \& Embrapa Pecuária Sul: Bovinocultura de Corte no Rio Grande do Sul, 3(1).

Núcleo de Estudos em Sistemas de Produção de Bovinos de Corte e Cadeia Produtiva (NESPRO) \& Empresa Brasileira de Pesquisa Agropecuária (EMBRAPA). (2018). Informativo NESPRO \& Embrapa Pecuária Sul: Bovinocultura de Corte no Rio Grande do Sul, 4(1).

Pereira, C. H., Patino, H. O., Hoshide, A. K., Abreu, D. C., Rotz, C. A. \&Nabinger, C. (2018). Grazing supplementation and crop diversification benefits for southern Brazil beef: A case study. Agricultural Systems. 162, 1-9.

Pereira, A. S., Shitsuka, D. M., Parreira, F. J., \&Shitsuka, R. (2018). Methodology of cientific research. UFSM. https://repositorio.ufsm.br/bitstream/hand le/1/15824/Lic_Com putacao_Metodologia-Pesquisa-Cientifica.pdf?sequence=1.

Silva, L. R. (2019). Simulação de cenários produtivos e econômicos em sistemas de recria-engorda de bovinos de corte. Dissertação de Mestrado em Ciências, Programa de Pós-graduação em Zootecnia, Universidade Federal de Pelotas, Pelotas. 